\title{
Percepción de los residentes sobre el impacto de la pandemia COVID 19 en la formación en profesionalismo
}

\author{
Residents'perspective of the impact of the COVID 19 pandemic \\ on professional competency training
}

\begin{abstract}
María Isabel González-Anglada' , Ana Isabel Huelmos-Rodrigo² , Fernando García-Pérez³ , Jesús Martín-Fernández4,
\end{abstract} Patricia Sanmartin-Fenollera ${ }^{5}$, Cristina Garmendia-Fernández ${ }^{6}$, Jesús Morán-Barrios ${ }^{7}$

\begin{abstract}
Resumen
Introducción: el profesionalismo es clave para construir la identidad de los profesionales sanitarios. El objetivo de este trabajo ha sido conocer en un colectivo de residentes, la influencia de la primera ola de la pandemia por SARS-CoV2 en sus competencias del profesionalismo. Materiales y métodos: estudio descriptivo transversal mediante cuestionario electrónico, remitido a 167 residentes, para medir su percepción sobre el impacto de la pandemia COVID-19 en el profesionalismo, con una escala verbal de cuatro niveles: excelente, por encima de lo esperado, lo esperado, por debajo de lo esperado. Se realizó, además, una pregunta abierta de "reflexión personal" analizada cualitativamente mediante un proceso de triangulación. La encuesta se hizo en el Hospital Universitario Fundación Alcorcón en los dos meses siguientes a la primera ola pandémica. Resultados: respondieron 59 residentes (35,3\%) de 21 especialidades. Sus lugares de trabajo fueron muy variados. Los atributos del profesionalismo valorados por encima de esperado o excelente fueron: trabajo en equipo $(74,6 \%)$, empatía $(71,2 \%)$ y responder a las necesidades del paciente por encima de las propias (69,5\%). Se encontró por debajo de lo esperado la gestión de las emociones (22\%). Discusión: la pandemia COVID-19 ha contribuido a reforzar la identidad profesional de los residentes, manifestándose a través de muchas dimensiones del profesionalismo. La gestión de las emociones fue la que obtuvo menor valoración.
\end{abstract}

Palabras clave: COVID-19; profesionalismo; educación médica; educación basada en competencias; residente.

\begin{abstract}
Introduction: Professionalism is essential to build the identity of health professionals. The aim in this study was to determine, in a group of residents, the influence of the first wave of SARS-CoV2 pandemic on their professionalism competencies. Methods: Cross-sectional descriptive study with an electronic survey sent to 167 residents of Hospital Universitario Fundación Alcorcón to assess their perception of the impact of COVID-19 pandemic on professionalism, with a four-level verbal scale: excellent, above expected, expected, below expected. In addition, an open-ended "personal reflection" question was qualitatively analyzed through a triangulation process. The survey was completed immediately after the first pandemic wave. Results: The questionnaire was answered by 59 residents (35.3\%) of 21 specialties from different workplaces. The attributes of professionalism rated above expected or excellent were: teamwork (74.6\%), empathy (71.2\%) and responding to patient's needs above their own (69.5\%). Management of emotions (21\%) was found to be below expectations. Discussion: COVID-19 pandemic has contributed to reinforce the professional identity of the residents, expressing itself through many dimensions of professionalism. Emotional management was the lowest rated.
\end{abstract}

Keywords: COVID-19; professionalism; education medical; competency-based education; clinical competence; resident.

Fecha de envío: 2021-06.15 - Fecha de aprobación: 2021-09-01

(1) Unidad de Docencia. Unidad de Medicina Interna. Hospital Universitario Fundación Alcorcón. Madrid. España

(2) Unidad de Cardiología. Hospital Universitario Fundación Alcorcón. Madrid. España

(3) Unidad de Rehabilitación. Hospital Universitario Fundación Alcorcón. Madrid. España

(4) Unidad Docente Multiprofesional de Atención Familiar y Comunitaria Oeste. Servicio Madrileño de Salud. Madrid. España

(5) Área de Farmacia. Hospital Universitario Fundación Alcorcón. Madrid. España

(6) Unidad de Medicina Interna. Hospital Universitario Fundación Alcorcón. Madrid. España

(7) Sociedad Española de Formación Sanitaria Especializada SEFSE-AREDA. Bilbao. España

Autor de correspondencia: mganglada@salud.madrid.org 


\section{Introducción}

El profesionalismo es un conjunto de principios y compromisos para lograr el bienestar de los pacientes y de la sociedad (ABIM Foundation ACP-ASIM Foundation and European Federation of Internal Medicine, 2002; Borrell-Carrio, et al, 2006), agrupa competencias clave en la formación de los profesionales sanitarios. Una tarea fundamental de los formadores es transmitir los valores profesionales (Morán-Barrios, et al, 2010). Existe gran debate sobre cómo aprender y evaluar el profesionalismo (Roberts, 2017).

El entorno formativo ha cambiado desde que la infección por coronavirus SARS-CoV-2 (COVID-19) fue reconocida como pandemia mundial. El sistema sanitario público ha sufrido un impacto sin precedentes (Soriano, 2020). En los hospitales se integraron todas las profesiones sanitarias, todas las especialidades y estamentos para afrontar la situación. La enfermedad ha afectado a millones de pacientes (Baena Díez et al., 2020), que han estado aislados, con la incertidumbre de una enfermedad grave y desconocida. En su atención los principios del profesionalismo han guiado a los sanitarios, abordando esta situación en circunstancias muy comprometidas (Harkin, 2021). Ha sido necesario considerar las necesidades de todos los pacientes en un entorno de gran incertidumbre (Harkin, 2020) con pocos recursos.

En el Hospital de referencia se multiplicó por tres la capacidad de atención entre febrero y abril de 2020. Los residentes interrumpieron sus rotaciones y abandonaron sus unidades de referencia para atender pacientes en diversos contextos sanitarios, con protección individual a veces inadecuada y elevado estrés. La pandemia obligó bruscamente, a modificar muchos aspectos relacionados con el proceso formativo de los residentes. Se ha alterado su formación, tanto la adquisición de competencias científico-técnicas como la menor supervisión (Millán, 2020). Se ha sugerido que la pandemia ha hecho resurgir un nuevo profesionalismo (Agarwal \& Gupta, 2020). Otras profesiones sanitarias la han reconocido como una oportunidad para reconstruir su identidad profesional (Li et al., 2020).

El trabajo tiene como objetivo conocer la influencia en el profesionalismo de la experiencia vivida durante la primera ola de la pandemia COVID-19 en un colectivo de residentes.

\section{Materiales y métodos}

Se realizó un estudio observacional descriptivo con los residentes del Hospital Universitario Fundación Alcorcón, que trabajaron desde el inicio de la pandemia COVID (entre el 2 de marzo y el 31 de mayo de 2020). En los dos meses posteriores se remitió un cuestionario electrónico de 15 ítems a 167 residentes, informándoles de su objetivo, la voluntariedad de su participación y del tratamiento anónimo de los datos. El comité de Ética de Investigación aprobó el estudio. Los datos se trataron conforme con el Reglamento de Protección de Datos (UE) 2016/679)(Diario Oficial de la Unión Europea, 2016) y la Ley Orgánica 3/2018 (Boletín Oficial del Estado, 2018). El formulario recogía datos demográficos, trabajo con pacientes COVID, suspensión del programa formativo, carga laboral, temor al contagio y síntomas COVID.

Los componentes del profesionalismo incluidos fueron: tres principios básicos (primacía del bienestar del paciente, autonomía y justicia social) y diez compromisos (competencia profesional, confidencialidad, honestidad, adecuadas relaciones con el paciente, calidad del cuidado, accesibilidad a los servicios de salud, distribución de recursos finitos, uso del conocimiento científico, manejo de conflictos de interés y responsabilidades profesionales) adaptados del American Board of Internal Medicine Foundation (ABIM Foundation ACP-ASIM Foundation and European Federation of Internal Medicine, 2002) y otros modelos (ABIM Foundation ACP-ASIM Foundation and European Federation of Internal Medicine, 2002; Comité Central. Instituto para la Educación Médica Internacional (IIME) New York. USA., 2002; Frank \& Danoff, 2007; Swing, 2007; Morán-Barrios, et al, 2010; Morán-Barrios, 2013). El cuestionario (parte de otro más amplio) se simplificó para garantizar una mayor participación. Al residente se le solicitó que valorara cómo se percibía respecto a cada atributo, tras la experiencia de la primera ola pandémica, mediante una escala verbal de cuatro niveles (excelente, por encima de lo esperado, lo esperado, por debajo de lo esperado). Se incluyó una pregunta abierta invitándole a realizar una reflexión narrativa desde su experiencia. Se realizó un análisis descriptivo de los diferentes atributos del profesionalismo. La pregunta abierta se analizó cualitativamente bajo el paradigma fenomenológico (Hamui, 2016), describiendo y discutiendo las reflexiones mediante un proceso de triangulación bajo criterios de adecuación, relevancia y validez (Calderón, 2002). Dichas reflexiones se agruparon según los diferentes atributos del formulario.

\section{Resultados}

Respondieron a la encuesta 59 residentes (35,3\%), 66,7\% mujeres. La tabla 1 muestra la distribución por ámbitos asistenciales y especialidades. La participación por año de residencia fue: 11,9\% primer año, $22 \%$ segundo, 30,5\% tercero, 20,3\% cuarto y $15,3 \%$ de quinto año. Todas las especialidades del hospital estuvieron representadas salvo cuatro.

El 91,5\% de los residentes manifestó haber trabajado con pacientes COVID, en urgencias (75,9\%), hospitalización $(48,1 \%)$, cuidados críticos (16,7\%), servicios centrales (9,3\%), centros de salud (18,5\%), hospital de campaña (3,7\%) y otros ámbitos (13\%). Algunos 
simultanearon varios lugares de trabajo. El 81,4\% suspendió la rotación que realizaba y el 71,2\% trabajó en su Unidad Docente.

El $76 \%$ consideró que la carga de trabajo fue mayor de la habitual y para el 11,9\% fue menor. El 80,7\% trabajó más de 40 horas semanales. Un 5,4\% hizo más de 7 guardias mensuales. El 3,6\% realizó 4 guardias, el 23,2\% 5, el 46,4\% 6, y el 21,4\% efectuó 7 guardias al mes.

El nivel de preocupación por contagiarse fue alto en el 28,8\% y bajo en el 27,1\%. Un 37,3\% presentó síntomas COVID.

La tabla 2 muestra los resultados de la percepción de su profesionalismo. Valoraron positivamente la mayoría de los atributos, destacando en el nivel por encima de lo esperado o excelente: trabajar en equipo eficazmente $(74,6 \%)$, ser empático con pacientes y familiares $(71,2 \%)$, dar respuesta a las necesidades del paciente por encima de las propias (69,5\%), presentar una adecuada conducta $(66,1 \%)$, proporcionar la más alta calidad de cuidados posibles $(61,1 \%)$, no discriminar por razones raciales, culturales, sociales, de edad, sexo o discapacidad (59,3\%), trabajar dentro de los límites (49,2\%), respetar la autonomía del paciente $(45,8 \%)$ autoevaluar la práctica clínica (45,8\%) y asumir el liderazgo (42,4\%). Las cualidades del profesionalismo en las que percibieron con más frecuencia estar por debajo de lo esperado fueron: gestionar las emociones (22\%), respetar la privacidad del paciente $(22 \%)$, trabajar según los principios de las organizaciones sanitarias (18,6\%), limitar el esfuerzo terapéutico (13,5\%) y facilitar el aprendizaje de los compañeros (10,2\%).

El análisis de las reflexiones sobre la experiencia vivida se sintetiza en la tabla 3 mostrando los fragmentos más relevantes de las respuestas clasificados según los atributos del profesionalismo.

Tabla 1: Distribución de los residentes que contestaron a la encuesta por ámbitos asistenciales y por especialidad.

\begin{tabular}{|l|l|}
\hline Ámbito asistencial & $\%$ \\
\hline Especialidades médicas & 52,7 \\
\hline Especialidades quirúrgicas & 22 \\
\hline Cuidados críticos (intensivos y anestesia) & 10,2 \\
\hline Servicios centrales & 5,1 \\
\hline
\end{tabular}

Tabla 2: Percepción de los residentes de sus competencias sobre el profesionalismo tras la experiencia en la primera ola de la pandemia.

\begin{tabular}{|c|c|c|c|c|c|c|}
\hline Componentes dentro de la competencia profesionalismo & \begin{tabular}{|l|}
$\begin{array}{l}\text { Excelente } \\
(\%)\end{array}$ \\
\end{tabular} & \begin{tabular}{|l|}
$\begin{array}{l}\text { Por encima de } \\
\text { lo esperado (\%) }\end{array}$ \\
\end{tabular} & \begin{tabular}{|l|}
$\begin{array}{l}\text { Lo esperado } \\
(\%)\end{array}$ \\
\end{tabular} & \begin{tabular}{|l|} 
Por debajo de \\
lo esperado (\%)
\end{tabular} & NA (\%) & $\begin{array}{ll}\mathbf{N} C \\
(\%)\end{array}$ \\
\hline $\begin{array}{l}\text { Proporcionar la más alta calidad de cuidados posibles con integridad, } \\
\text { honestidad, humanidad, altruismo y sentido del deber }\end{array}$ & 11,9 & 49,2 & 25,4 & 8,5 & 5 & --- \\
\hline Dar respuesta a las necesidades del paciente por encima del interés propio & 23,7 & 45,8 & 16,9 & 10,2 & 3,4 & --- \\
\hline Ser empático con los pacientes y las familias & 32,2 & 39 & 22 & 1,7 & 3,4 & 1,7 \\
\hline $\begin{array}{l}\text { Respetar la autonomía de los pacientes y ayudar a tomar decisiones } \\
\text { informadas }\end{array}$ & 6,8 & 39 & 40,7 & 8,5 & 5 & --- \\
\hline Limitar el esfuerzo terapéutico tras valorar el riesgo y los beneficios & 10,2 & 15,3 & 47,5 & 13,5 & 13,5 & --- \\
\hline Respetar la privacidad del paciente & 6,8 & 20,3 & 44,1 & 22 & 6,8 & --- \\
\hline $\begin{array}{l}\text { No discriminar por razones raciales, culturales, sociales, de edad, sexo } \\
\text { o discapacidad. }\end{array}$ & 40,7 & 18,6 & 32,2 & 3,4 & 5,1 & --- \\
\hline $\begin{array}{l}\text { Trabajar dentro de los límites de las propias capacidades y pedir ayuda } \\
\text { cuando es necesario }\end{array}$ & 13,6 & 35,6 & 37,2 & 8,5 & 3,4 & 1,7 \\
\hline Trabajar en equipo de forma eficaz & 27,1 & 47,5 & 18,6 & 3,4 & 3,4 & --- \\
\hline $\begin{array}{l}\text { Asumir el liderazgo cuando la situación lo requiere y distribuir las cargas } \\
\text { de trabajo con los compañeros }\end{array}$ & 10,2 & 32,2 & 37,2 & 10,2 & 10,2 & --- \\
\hline $\begin{array}{l}\text { Mostrar una adecuada conducta personal e interpersonal en el entorno } \\
\text { de trabajo }\end{array}$ & 8,5 & 57,6 & 25,4 & 5,1 & 3,4 & --- \\
\hline Autoevaluar la práctica clínica y cambiar comportamientos & 5,1 & 40,7 & 42,3 & 6,8 & 5,1 & --- \\
\hline $\begin{array}{l}\text { Gestionar adecuadamente las propias emociones a través de una } \\
\text { retroalimentación positiva }\end{array}$ & 3,4 & 23,7 & 45,8 & 22 & 3,4 & 1,7 \\
\hline Facilitar el aprendizaje de los compañeros & 1,7 & 25,4 & 55,9 & 10,2 & 6,8 & --- \\
\hline $\begin{array}{l}\text { Trabajar acorde a los principios de las organizaciones sanitaras, funda- } \\
\text { mentos económicos y legales }\end{array}$ & 0 & 20,3 & 52,6 & 18,6 & 8,5 & --- \\
\hline
\end{tabular}

$\mathrm{NA}=$ No aplica; $\mathrm{NC}=$ No contesta 
Tabla 3: Reflexiones más relevantes de los residentes relacionados con los diferentes atributos del profesionalismo.

\begin{tabular}{l}
\hline Competencia profesionalismo \\
Proporcionar la más alta calidad de cuidados posibles \\
con integridad, honestidad, humanidad, altruismo y \\
sentido del deber.
\end{tabular}

Dar respuesta a las necesidades del paciente por encima del interés propio.

Ser empático con los pacientes y las familias.

Respetar la autonomía de los pacientes y ayudar a
tomar decisiones informadas.

Limitar el esfuerzo terapéutico tras valorar el riesgo y los beneficios.

Respetar la privacidad del paciente.

No discriminar por razones raciales, culturales, sociales, de edad, sexo o discapacidad.

Trabajar dentro de los límites de las propias capacidades y pedir ayuda cuando es necesario.

Trabajar en equipo de forma eficaz.

Asumir el liderazgo cuando la situación lo requiere y distribuir las cargas de trabajo con los compañeros

Mostrar una adecuada conducta personal e interpersonal en el entorno de trabajo.

Autoevaluar la práctica clínica y cambiar comportamientos.

Gestionar adecuadamente las propias emociones a través de una retroalimentación positiva.

Facilitar el aprendizaje de los compañeros.

Trabajar acorde a los principios de las organizaciones sanitaras, fundamentos económicos y legales.

\section{Frases más relevantes}

"La pandemia ha sido un tsunamique nos ha pasado a todos por encima. Aun así, estoy muy orgulloso de mis adjuntos y todos mis compañeros por la entereza, el profesionalismo, la capacidad de trabajar en equipo y, sobre todo, la de improvisar por el bien de nuestros pacientes"

"La mayor enseñanza ha sido la empatía con el paciente, la información a las familias y la calidad en la atención que debemos ofrecer".

"La situación ha puesto de relieve, por una parte, el compromiso ético y profesionalidad de los sanitarios, pero también la escasez de medios y recursos humanos y materiales"

"Los meses de pandemia han supuesto un esfuerzo sobrehumano para todos los profesionales del hospital y pese a que esto ha influido en la formación de los residentes no había otra opción más que sumarnos a ayudar y donde hemos aprendido otros aspectos importantes de la medicina".

"He intentado formarme e investigar, y sobre todo empatizar e informar a familiares de pacientes ingresados"

"El periodo COVID ha traído aspectos negativos, pero he podido ver lo mejor de mis compañeros y de mi misma: empatía, trabajo en equipo, comunicación intra e interhospitalaria, capacidad de superación".

"Comunicación con el paciente y manejo de la incertidumbre de la situación e información" "El trato psicológico de un paciente es tan importante como el médico en los momentos de gravedad". "Asumir las limitaciones de la medicina. Velar por el cuidado de los pacientes en el fin de vida" "La mayor enseñanza el trabajo en equipo, la capacidad de sacrificio de las personas, no dar tratamiento si no está justificado".

"Lo peor era pensar no abarcar a tratar a las personas con dignidad y los medios adecuados"

"Me he sentido superada por el acúmulo de pacientes en la urgencia para ingreso, sin camas disponibles, ubicados en sillones -sillas en el pasillo en el mejor de los casos, y en el peor de los casos en el suelo con una sábana, con saturaciones bajas de oxígeno".

"La pandemia COVID ha causado una repercusión sin precedentes y una alteración severa del manejo intrahospitalario de pacientes no COVID, con el aumento de tiempo por el cribado ictus y ausencia de personal enfermero cualificado, se produjeron pérdidas de indicación de tratamiento en el ictus". "He explorado nuevas formas de resolver problemas a los pacientes. He asumido riesgos y me ha hecho salir más segura de mí misma. He aprendido cada día. Me ha costado gestionar la tensión con otros compañeros ante los cambios continuos y el miedo"

"Me he sentido superada por el desconocimiento de patologías del paciente adulto".

"Considero que ha sido una experiencia vital y profesional con situaciones muy complejas y duras.

El trabajo en equipo y el humanismo de mis compañeros ha sido enriquecedor"

"La mayor enseñanza de este periodo ha sido el trabajo en equipo y el compañerismo".

"Me he dado cuenta de lo importante que es un buen liderazgo y una buena organización para

el trabajo en situaciones difíciles salga adelante. También me reafirmo en lo importante que es ser humilde y pedir ayuda o reconocer los errores".

"He tenido que adaptarme rápido y constantemente a los cambios y protocolos de las diferentes unidades. También he tenido que tomar muchas decisiones sola"

"Lo más importante mi capacidad de resiliencia".

"He aprendido a ser muchísimo más autónoma y a tomar decisiones en el manejo de pacientes" "La experiencia vivida supera cualquier expectativa que pudiera tener previa a cualquier rotación y cada día sigue siendo de nuevo aprendizaje".

"He aprendido a gestionar el estrés psicológico y laboral que me he ido encontrando. Mis compañeros estaban muy saturados y he tenido que aprender a gestionar yo sola"

"Una etapa de mucho estrés y ansiedad con conflictos emocionales y éticos a diario"

"El estrés emocional al vivir con pacientes críticos que no podían optar a cuidados intensivos o a la soledad de los pacientes en el aislamiento".

"Hemos cosechado un aprendizaje inconmensurable sobre nosotros mismos, la enfermedad, la humanidad, el trato con el paciente y sus familiares, la red de apoyo entre compañeros, el trabajo en equipo, el liderazgo.

"Hemos intentado responder de la mejor forma que nos permitieron el diseño de nuestro sistema y nuestras propias aptitudes a un evento que nos desbordó completamente muy rápidamente" "Creo que ha sido una experiencia muy dura pero de la que se pueden sacar multitud de aprendizajes, ha sido conocer la medicina bajo escasez de recursos, algo que no habíamos experimentado previamente, lo más positivo ha sido el trabajo en equipo, lo peor, la impotencia y la sobrecarga de trabajo y emocional". 


\section{Discusión}

La primera ola de la pandemia COVID-19 nos enfrentó a una enfermedad contagiosa, desconocida, grave, sin tratamiento etiológico y con un rápido incremento de pacientes que colapsó los hospitales. En nuestro hospital creció un 300\% la capacidad de hospitalización, urgencias e intensivos con nuevos espacios. Los residentes dejaron sus rotaciones y formaron parte de los equipos COVID, abandonando sus itinerarios formativos y cualquier otra formación complementaria planificada previamente. La atención de pacientes graves, aislados y con escasos recursos les hizo afrontar decisiones difíciles. La muestra de personas que respondió la encuesta es un grupo feminizado, en la tercera década de la vida, como ocurre en el conjunto del colectivo, y que sufrió una enorme sobrecarga de trabajo y emocional. Esto ha potenciado el desarrollo de su identidad profesional, la empatía, el altruismo, anteponer las necesidades del paciente, el trabajo en equipo y la conducta adecuada. La gestión de las emociones fue el aspecto menos fortalecido (tabla 2).

Durante la pandemia todos los principios y compromisos del profesionalismo han tendido una gran relevancia como se reseña a continuación.

Principio de primacía del bienestar del paciente. La responsabilidad profesional de los residentes ante la grave situación ha quedado patente. Más del $60 \%$ se ha sentido reforzado positivamente (nivel excelente o por encima de lo esperado) en los atributos "dar respuesta a las necesidades del paciente", "proporcionando la más alta calidad de cuidados" (tabla 2). Han vivido una situación extrema, asumiendo una elevada carga asistencial y menor seguridad para su salud: "La pandemia ha sido un tsunami..." (tabla 3). En la enseñanza del profesionalismo, eminentemente práctica, tiene especial relevancia la función de modelo (Roberts, 2017). Durante la pandemia han emergido actitudes profesionales que han servido como ejemplo.

Principio de la autonomía del paciente. Un 45,8\% de los residentes dice haber respetado la autonomía del paciente de forma destacada. Tratar pacientes con una enfermedad de elevada mortalidad, ha enfrentado a los residentes a asumir que conocimiento y tecnología no son suficientes para una buena práctica clínica: "El trato psicológico de un paciente es tan importante como el médico..." (tabla 3) y no ofrecer tratamientos fútiles explicando con respeto y sinceridad el motivo de la prescripción, valorando riesgos y beneficios y limitando el esfuerzo terapéutico. Ante la gravedad de la enfermedad, la soledad del paciente aislado y la gestión emocional con las familias mediante información telefónica, los residentes han desarrollado la compasión como guía de acción. Se han ejercitado en comunicar con honestidad y mostrar empatía, aspecto crucial para una atención sanitaria eficaz (Halpern \& Douglas, 2020): "He intentado formarme e investigar,...empatizar..." (tabla 3).

Principio de la justicia social. El 59,3\% de los residentes afirma no haber discriminado la atención a pacientes por ningún motivo. Durante esta pandemia los pacientes de edad avanzada y los que asociaban comorbilidades eran los más vulnerables y con menos posibilidades de sobrevivir: “...conocer la medicina bajo escasez de recursos..." (tabla 3). En la primera ola los enfermos con elevadas necesidades ventilatorias superaban los recursos existentes. Por ello fue necesario crear comités multidisciplinares que priorizaran su asignación. Sin embargo, determinar que un paciente realice un tratamiento en detrimento de otro ha creado dilemas éticos (Fritz et al., 2020). La integridad en usar el conocimiento y optimizar los recursos deben guiar las acciones. Según los residentes estas situaciones les han producido gran estrés emocional, con conflictos éticos, al tratar con pacientes críticos que no podían recibir cuidados intensivos. A pesar del alto porcentaje de residentes que consideraban haber gestionado adecuadamente sus emociones mediante retroalimentación positiva, un 22\% manifiestan no haber podido.

Compromisos profesionales. Compromisos profesionales. La pandemia obligó a crear equipos heterogéneos. Esto conlleva una identificación previa de perfiles y experiencias (Martínez-Pillado et al., 2021). El trabajo en equipo ha permitido dar respuestas inmediatas en distintos escenarios. El 74,6\% de residentes consideró haber trabajado eficazmente en equipo. En situaciones difíciles sentirse parte del equipo ayuda a afrontar la adversidad: "El trabajo en equipo y el humanismo... ha sido enriquecedor". El humanismo se ha manifestado entre profesionales (palabras de aliento de un compañero) y hacia pacientes (conectarle telemáticamente con sus familiares, remitiéndole cartas...). La pandemia enseñó a olvidar el ego, asumir liderazgos inesperados, reconocer errores, aprender y mejorar (Castells, 2020). Aprendizaje, en todos los sentidos, ha sido la palabra más repetida: "Aprendizaje inconmensurable..." (tabla 3). La disposición a actuar correctamente, el aprendizaje con el paciente y con el equipo han podido modelar positivamente su identidad. Según Victoria Camps "la adquisición de las virtudes respeto, sinceridad, compasión, olvido de sí, hace que los principios de beneficencia, autonomía, justicia y cuidados de salud no sean solo bellas palabras de las guías de buenas prácticas, sino prácticas reales incorporadas a la actividad profesional" (Camps, 2015). Nuestros resultados lo apoyan.

Una importante fortaleza de nuestro trabajo es su originalidad. No encontramos publicaciones sobre el impacto de la pandemia en el profesionalismo de los residentes. Resaltamos la relevancia de la información (Calderón, 2002) y que estuvieran altamente 
representadas las especialidades. Señalamos, como limitación, el ajustado porcentaje de respondedores (es posible que un mayor impacto emocional fuera un estímulo para contestar la encuesta). Los instrumentos estandarizados para evaluar el profesionalismo son muy extensos, no se utilizaron para mejorar la tasa de respuestas y el formulario formaba parte de otro estudio más amplio. Nuestros resultados no son generalizables.

\section{Conclusión}

La pandemia COVID-19 ha enfrentado a los residentes con dilemas complejos en los que han aplicado virtudes y principios éticos de la profesión sanitaria percibiendo su influencia positiva en la construcción de los valores profesionales.

El diseño de los programas formativos que ya contemplaba la construcción de estos valores profesionales, debería incluir herramientas que pudiesen ser utilizadas en situaciones de excepcionalidad, pues éstas no limitan la necesidad de esta tarea, sino que la hacen aún más importante.

\section{Referencias}

ABIM Foundation ACP-ASIM Foundation and European Federation of Internal Medicine (2002) Medical Professionalism in the New Millennium: A Physician Charter. Annals of Internal Medicine 136, 243-246.

Agarwal P. \& Gupta V. (2020) COVID 19 pandemic: An opportunity to investigate medical professionalism. Canadian Medical Education Journal 12; e103-e104.

Baena Díez JM, Barroso M, Cordeiro-Coelho SI, Díaz JL. \& Grau M. (2020). Impact of COVID-19 outbreak by income: hitting hardest the most deprived. Journal of Public Health 42, 698-703.

Boletín Oficial del Estado (2018) Ley Orgánica 3/2018, de 5 de diciembre, de Protección de Datos Personales y garantía de los derechos digitales., Boletin Oficial del Estado. Accedido en https://www.boe.es/ buscar/doc.php?id=BOE-A-2018-16673 el 12 de febrebro de 2021.

Borrell-Carrio, F., Epstein, R. M. and Pardell Alentà, H. (2006). Profesionalidad y professionalism: fundamentos, contenidos, praxis y docencia. Medicina Clínica 127, 337-342.

Calderón C. (2002). Quality criteria in Qualitative Research in Health: notes for a necessary debate. Revista espanola de salud publica 76, pp.473-82.

Camps V. (2015). Los valores éticos de la profesión sanitaria. Educación Médica 16, 3-8.
Castells A. (2020). COVID-19: A pandemic of values. Gastroenterología y Hepatología (English Edition) 43, 329-330.

Comité Central. Instituto para la Educación Médica Internacional (IIME) New York. USA. (2002). Requisitos Globales Minimos En Educación Médica. Educación Médica, (1), pp. 11-19.

Diario Oficial de la Unión Europea (2016) Reglamento (UE) 2016/679 del Parlamento Europeo y del Consejo de 27 de Abril de 2016, relativo a la protección de las personas físicas en lo que respecta al tratamiento de datos personales y a la libre circulación de estos datos y por el que se deroga la Directiva 95/46/CE (Reglamento general de protección de datos) , Diario Oficial de la Unión Europea. Accedido en https://eur-lex. europa.eu/legal-content/ES/TXT/?uri=CELEX\%3A32016R0679\&qid=1630445068409 el 28 de abril de 2021.

Frank JR. \& Danoff D. (2007) 'The CanMEDS initiative: implementing an outcomes-based framework of physician competencies. Medical Teacher 29, 642-647.

Fritz Z, Huxtable R, Ives J, Paton A, Slowther AM. \& Wilkinson D. (2020).Ethical road map through the covid-19 pandemic. BMJ 21;369:m2033.

Halpern J. \& Douglas JO. (2020) Sustaining Clinical Empathy During the Pandemic, The Hastings Center. Accedido en https://www. thehastingscenter.org/sustaining-clinical-empathy-during-the-pandemic/ el 12 de febrero de 2021.

Hamui Sutton A. (2016). La pregunta de investigación en los estudios cualitativos, Investigación en Educación Médica 5, 49-54.

Harkin D. (2021). COVID-19 and medical professionalism in a pandemic. Postgraduate Medical Journal 97, 53-54.

Harkin DW. (2020). Covid-19: Balancing personal risk and professional duty The BMJ 29;369:m1606.

Li Z, Zuo Q, Cheng J, Zhou Y, Li Y, Zhu L. \& Jiang X. (2020). Coronavirus disease 2019 pandemic promotes the sense of professional identity among nurses. Nursing Outlook 69: 389-398.

Martínez-Pillado M, Villalobos Hidalgo J, del Llano-Señarís J. \& Varela Duránda M. (2021). Los valores relacionados con la formación de profesionales sanitarios en un entorno COVID-19. Educación Médica 22, S16-S18.

Millán Núñez Cortés J. (2020). COVID-19 por SARS-Cov2 también ha afectado a la Educación Médica. Educación Médica 21, 261-264. 
González-Anglada et al.

Morán- Barrios J. (2013). Un nuevo profesional para una nueva sociedad. Respuestas desde la educación médica: la formación basada en competencias. Revista de la Asociación Española de Neuropsiquiatría 33, 385-405.

Morán- Barrios J, Ruiz de Gauna Bahillo P. \& Miembros de la Junta Directiva de la Sociedad de Educación Médica de Euskadi. (2010). Reinventing specialty training of physicians? Principles and challenges. Nefrologia 30, 604-12.
Roberts TE. (2017). Enseñar, aprender y evaluar el profesionalismo: el mayor reto de todos. Revista de la Fundación Educación Médica 20, 47.

Soriano JB. (2020). Medicina, Epidemiología y Humanismo antes y después de la COVID-19'. Revista Clínica Española 220, 503-506.

Swing SR. (2007). The ACGME outcome project: retrospective and prospective. Medical Teacher 29, 648-654. 